SCIENCE CHINA

Physics, Mechanics \& Astronomy

\title{
Quasi-static motion of microparticles at the depinning contact line of an evaporating droplet on PDMS surface
}

\author{
Ying-Song $\mathrm{Yu}^{1 *}$, Xue-Lian Xia ${ }^{1}, \mathrm{Xu}$ Zheng ${ }^{2}$, Xianfu Huang ${ }^{2,3}$, and Jin-Zhi Zhou ${ }^{1}$ \\ ${ }^{1}$ Department of Mechanics, School of Civil Architecture and Environment, Hubei University of Technology, Wuhan 430068, China; \\ ${ }^{2}$ State Key Laboratory of Nonlinear Mechanics, Institute of Mechanics, Chinese Academy of Sciences, Beijing 100190, China; \\ ${ }^{3}$ School of Engineering Science, University of Chinese Academy of Sciences, Beijing 100049, China
}

Received April 17, 2017; accepted May 16, 2017; published online July 14, 2017

\begin{abstract}
In this paper, evaporation of sessile water droplets containing fluorescent polystyrene (PS) microparticles on polydimethylsiloxane (PDMS) surfaces with different curing ratios was studied experimentally using laser confocal microscopy. At the beginning, there were some microparticles located at the contact line and some microparticles moved towards the line. Due to contact angle hysteresis, at first both the contact line and the microparticles were pinned. With the depinning contact line, the microparticles moved together spontaneously. Using the software ImageJ, the location of contact lines at different time were acquired and the circle centers and radii of the contact lines were obtained via the least square method. Then the average distance of two neighbor contact lines at a certain time interval was obtained to characterize the motion of the contact line. Fitting the distance-time curve at the depinning contact line stage with polynomials and differentiating the polynomials with time, we obtained the velocity and acceleration of both the contact line and the microparticles located at the line. The velocity and the maximum acceleration were, respectively, of the orders of $1 \mu \mathrm{m} / \mathrm{s}$ and $20-200 \mathrm{~nm} / \mathrm{s}^{2}$, indicating that the motion of the microparticles located at the depinning contact line was quasi-static. Finally, we presented a theoretical model to describe the quasi-static process, which may help in understanding both self-pinning and depinning of microparticles.
\end{abstract}

droplet, evaporation, PDMS, contact line, van der Waals force, electrostatic force, drag force, capillary force

PACS number(s): 68.08.-p, 68.03Fg, 82.70.Dd

Citation: Y. S. Yu, X. L. Xia, X. Zheng, X. Huang, and J. Z. Zhou, Quasi-static motion of microparticles at the depinning contact line of an evaporating droplet on PDMS surfaces, Sci. China-Phys. Mech. Astron. 60, 094612 (2017), doi: 10.1007/s11433-017-9060-3

\section{Introduction}

When droplets of colloidal suspensions and solutions of nonvolatile species evaporate on a solid surface, ring-like solid residues will usually be left after evaporation, which is commonly known as coffee-ring phenomenon. Deegan et al. [1] pointed out that it is the pinning of the three-phase contact line that induces the formation of the coffee stain. Later, $\mathrm{Hu}$ and Larson [2] conducted evaporation of octane droplets

*Corresponding author (email: yuystm@mail.hbut.edu.cn) containing polymethyl methacrylate (PMMA) particles on glass surface and found that suppression of Marangoni flow is also necessary for the formation of the coffee ring. This coffee-ring effect (reviewed in [3,4]) will limit the application of droplet evaporation in many fields such as printing, biology and complex assembly. To obtain a uniform evaporation stain, a lot of methods have been proposed such as by changing the shape of microparticles [5], using electrowetting [6,7], adding surfactants [8] or hydrosoluble polymers [9], promoting particle adsorption and long-range interaction [10], changing the evaporation environment [11] and using contact angle hysteresis [12]. 
To better apply evaporative-induced self-assembly [13], understanding the mechanism of self-pinning is necessary. During the latest years, a lot of researchers focused on this problem. Weon et al. [14] studied evaporation of liquid suspension of colloidal particles with different radii and found that capillary force will repel the coffee-ring effect. Jung et al. [15] compared the magnitudes of various forces including the drag, electrostatic, van der Waals, and capillary forces using the scaling analysis to elucidate the impact of these forces on the particle motion. They found that i) the motion of a single particle suspended in liquid is mostly affected by drag force when the contact line is pinned, and ii) later, with the incidence of the contact line recession, the capillary force takes over the control of the single particle motion. Wong et al. [16] elucidated the physics of particle separation during coffee-ring formation, which is based on a particle-size selection mechanism near the contact line of an evaporating droplet. In 2011, based on the studies by Jung et al. [15] and Wong et al. [16], Chhasatia and Sun [17] set up a self-pinning mechanism (note that in this paper, they regarded that the velocity of water in evaporating drop was $0.2 \mathrm{~m} / \mathrm{s}$. However, at room temperature, the velocity is of the order of $1 \mu \mathrm{m} / \mathrm{s}$ [18]). Later, Weon and Je [18] studied the differences in spreading and drying behaviors of pure and colloidal droplets using optical and confocal imaging methods, and proposed a self-pinning mechanism based on spreading inhibition by colloids. Bhardwaj et al. [19] found that the $\mathrm{pH}$ of the solution influences the dried deposit pattern, explained the transition between these patterns by considering how Derjaguin-Landau-Verwey-Overbeek (DLVO) interactions such as the electrostatic and van der Waals forces modify the particle deposition process, and proposed a phase diagram to describe the influence of flow patterns on the shape of a colloidal deposit. Yuan et al. $[20,21]$ elegantly proposed a combined theoretical framework in which both viscous resistance and molecular friction in the triple-phase region take place. The energy dissipation near the contact line might be a significant factor for self-pinning of microparticles. Using molecular dynamic simulation, Li et al. [22] predicted three styles of contact line motion including complete slipping, alternate pinning-depinning, and complete pinning. Different from Jung et al. [15], Wong et al. [16], Weon et al. [18] and Chhasatia and Sun [17], the capillary force is supposed to act on the particle along a declined redirection rather than parallel to the solid-liquid interface $[23,24]$. Combining all the above studies, we aim at setting up a novel self-pinning mechanism. Besides, moving contact line problem $[25,26]$ is an important issue of droplet evaporation and it consists of 7-8 length and time scales from the atomistic to the continuum. In the microscale region, both the van der Waals interactions and nanoscale viscous dissipation play important roles [27].
To set up the new mechanism, we experimentally studied evaporation of liquid suspension of fluorescent polystyrene (PS) microparticles on polydimethylsiloxane (PDMS) surfaces. PDMS has been widely used in micro- and nanosystems due to its good biocompatibility, nontoxicity, optical transparency and ease of fabrication. Recently, researches on wettability of PDMS surface [28-33] have been reported, indicating that the substrate elasticity has a significant influence on wetting and evaporation characteristics as well as the formation of evaporation stains. In this paper, using particle tracking velocimetry technique (PTV) [34], we focused on the evolution of contact line and the microparticles located at the line. We found that at first there were some microparticles located at the contact line, which are self-pinned at the pinned line, and then they moved spontaneously with the depinning contact line. Using the least square method, we calculated the moving distance of the depinning contact line and obtained its velocity and acceleration. The velocity and maximum acceleration were, respectively, of the order of $1 \mu \mathrm{m} / \mathrm{s}$ and $20-200 \mathrm{~nm} / \mathrm{s}^{2}$, indicating that the motion of microparticles located at the depinning contact line was quasi-static. Later, a novel mechanism for the quasi-static process was proposed from the view of force equilibrium.

\section{Experiments}

We prepared PDMS (Sylgard 184, Dow Corning, USA) membrane for studying droplet evaporation. PDMS (the ratios of base to curing are, respectively, 5:1, 10:1 and 20:1) was stirred fully and degassed for $15 \mathrm{~min}$, and then spin-coated on clean glass at the speeds of $500 \mathrm{r} / \mathrm{min}$ for $20 \mathrm{~s}$ and then $1000 \mathrm{r} / \mathrm{min}$ for $30 \mathrm{~s}$. Finally the samples were heated on a hot-plate at $150^{\circ} \mathrm{C}$ for $30 \mathrm{~min}$. To study the characteristics of the depinning contact line of evaporating droplets, $1.0 \mu \mathrm{m}$-diameter Fluo-Max ${ }^{\circledR}$ fluorescent green PS microparticle suspension (initial concentration: $1 \mathrm{wt} . \%$ ) was diluted to $6.25 \times 10^{-4}$ wt. $\%$ with deionized water. The diluted suspension was ultrasonically stirred for $10 \mathrm{~min}$ to ensure microparticles were homogenously dispersed. $0.6 \mu \mathrm{L}$ suspension was extracted using a micropipettor and then deposited slightly on PDMS surfaces. Before deposition, we adjusted the local confocal microscopy (Yokogawa CSU-1, Japan) to emit a blue laser with a wavelength of $488 \mathrm{~nm}$, which was used to cause the microparticles to fluoresce at $508 \mathrm{~nm}$. Once a droplet was deposited on PDMS surfaces, the microscopy was adjusted to focus on the solid-liquid interface and recorded the evaporation process at $5 \mathrm{fps}$, as shown in Figure 1. The environmental temperature and relative humidity were, respectively, $(24.6 \pm 1)^{\circ} \mathrm{C}$ and $37 \% \pm 1 \%$. To ensure the experiments reproducible, each experiment was repeated six times. 


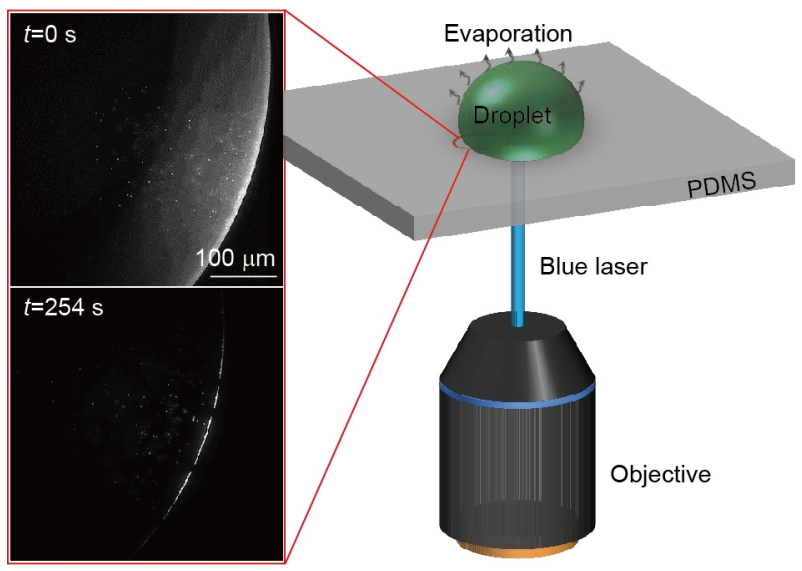

Figure 1 (Color online) Scheme of the set-up for sessile droplet evaporation.

\section{Result and discussion}

Figure 2 shows the images of the solid-liquid interface of evaporating droplets on PDMS surface (10:1) at different time. Using the software ImageJ, we obtained the pixel values of the fluorescent microparticles at the contact lines and converted them into values in unit of $\mu \mathrm{m}$. Eight or nine microparticles at each line were chosen and analyzed, as shown in Figure 3. Theoretically, every contact line was a circle-like arc. Therefore, we obtained the coordinates of the circle centers and the radii using the least square method [35], and found that the contact lines were all circle-like with errors less than $0.6 \%$. To determine the moving distance of the contact line at a time interval, we calculated the distance $d_{j}$ of each of the points at time $t_{i}$ to the neighbor contact line at time $t_{i+1}$ as $d_{j}=\sqrt{\left(x_{j}{ }^{i}-x_{0}{ }^{i+1}\right)^{2}+\left(y_{j}{ }^{i}-y_{0}{ }^{i+1}\right)^{2}}-r^{i+1}$, where $\left(x_{j}{ }^{i}, y_{j}{ }^{i}\right)$ was the coordinates of the $j$-th microparticle at the contact line at time $t_{i},\left(x_{0}{ }^{i+1}, y_{0}{ }^{i+1}\right)$ and $r^{i+1}$ were the coordinates of the center and radius of the line at time $t_{i+1}$, respectively. $N$ was the total number of the selected points at a contact line. The average distance between the $i$-th and $(i+1)$-th contact lines was obtained as $d^{i}=\frac{1}{N} \sum_{j=1}^{N} d_{j}$. Then the distance between the initial contact line and the $i$-th contact line was written as $D=\sum_{j=0}^{i-1} d^{j}$.

Figure 4 shows the moving distance of the contact line versus time, indicating that evaporation proceeds with constant contact radius mode due to contact angle hysteresis as well as surface deformation induced by droplet, etc., and then with the moving contact line. In this paper, we focus on the depinning contact line and the motion of the microparticles located at the line. Using the least square method, we fitted the curves at the depinning contact line stage with polynomials (Figure 5) as $D=\sum_{k=0}^{N} a_{k} t^{k}, t \in\left[t_{0}, t_{1}\right]$. Differentiating the

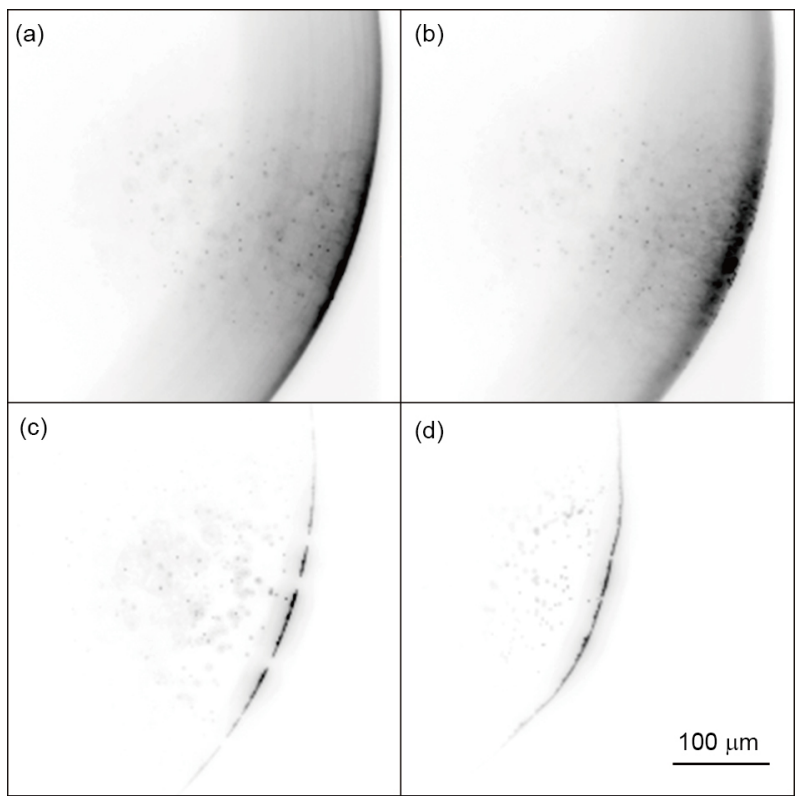

Figure 2 Images of solid-liquid interface of evaporating droplet on 10:1 PDMS surface. (a) $t=0 \mathrm{~s}$; (b) $t=52 \mathrm{~s}$; (c) $t=254 \mathrm{~s}$; (d) $t=320 \mathrm{~s}$.

polynomials with time, we obtained the velocity and the acceleration of the line.

Figure 6 shows the velocity and acceleration of the contact lines of evaporating droplets on different PDMS surfaces, respectively. From the figures, we found that the velocity and the maximum acceleration were of the order $1 \mu \mathrm{m} / \mathrm{s}$ and 20-200 nm/ $\mathrm{s}^{2}$, respectively. Moreover, at the final stage, the velocity increases more greatly for 10:1 and 20:1 than for 5:1, and the acceleration for 5:1 increases slightly while that for 10:1 increases greatly and it changes from positive to negative for 20:1.

From Figure 2(c) and (d), we can find that at the depinning contact line stage, there are one or several (no more than 3 ) microparticles located at the contact line along the radial direction. The density of the microparticle is $1050 \mathrm{~kg} / \mathrm{m}^{3}$, then the mass of a microparticle is $m_{0}=5.5 \times 10^{-16} \mathrm{~kg}$. Using Newton's second law, the resultant force acting on the microparticles along the radial direction $\sum F=n m_{0} a$ is of the order $\left(10^{-9}-10^{-10} \mathrm{pN}\right)$, here $n$ is the number of microparticles at the depinning contact line along radial direction.

Microparticles inside an evaporating droplet experience several forces, viz., van der Waals force, electrostatic force, drag force and capillary force. The van der Waals force between a microparticle and the substrate in a fluid medium is given by $[15-17,36]$ :

$F_{\text {wps }}=\frac{2 A_{132} R^{3}}{3 z^{2}(z+2 R)^{2}}$,

where $A_{132}=\left(\sqrt{A_{11}}-\sqrt{A_{33}}\right)\left(\sqrt{A_{22}}-\sqrt{A_{33}}\right)$ is the Hamaker 

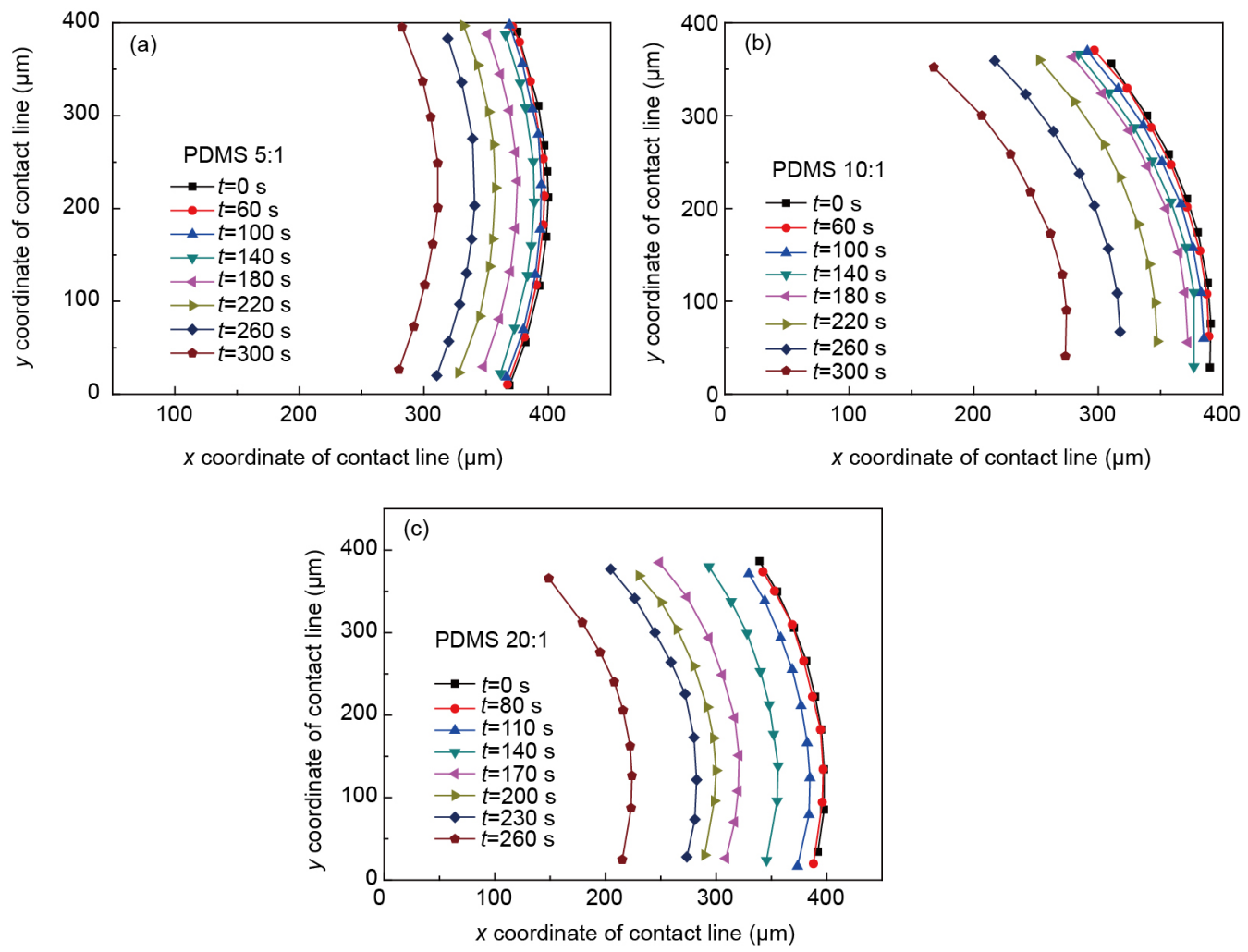

Figure 3 (Color online) Contact line of evaporating droplet on PDMS surfaces. (a) 5:1; (b) 10:1; (c) 20:1.

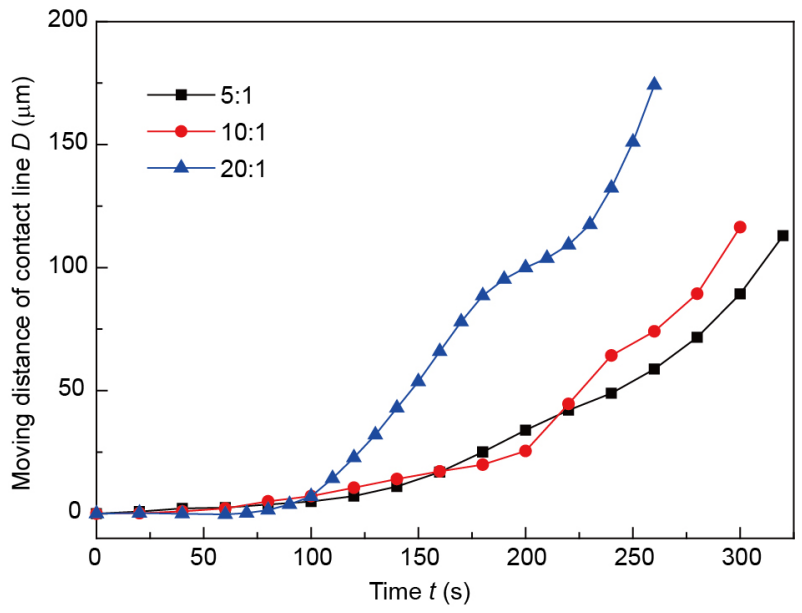

Figure 4 (Color online) Distance between the initial and current contact lines.

constant, the subscripts 1,2 and 3 denote the microparticle, the substrate and the fluid medium, respectively. $A_{i i}$ is the Hamaker constant of two bodies of material $i$. $z$ is the particle-substrate separation distance. $R$ is the radius of a microparticle. When $z$ is far less than $R$, the force can be simplified as:

$F_{\text {wps }}=\frac{A_{132} R}{6 z^{2}}$.

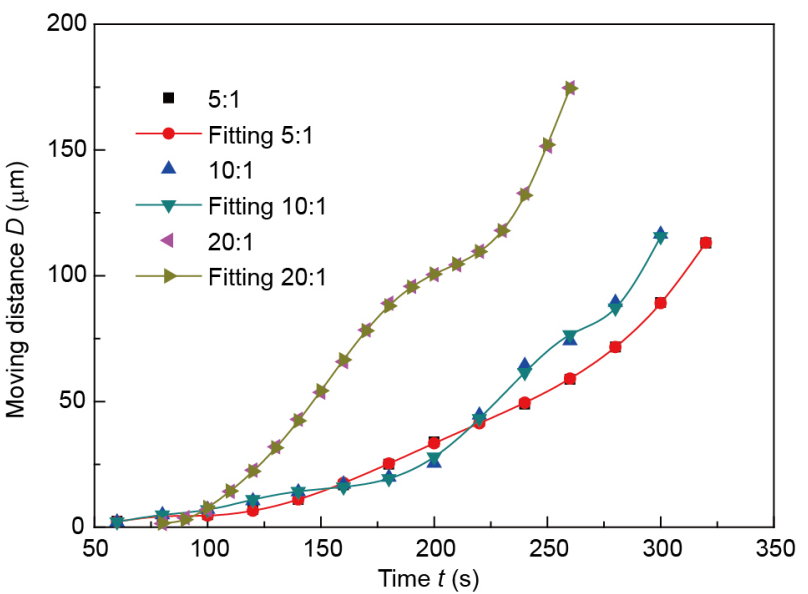

Figure 5 (Color online) Fitting of moving distance of contact line.

The van der Waals force between microparticles in a fluid is given by $[15-17,36]$ :

$F_{\text {wpp }}=\frac{A_{131} R}{12 z_{1}^{2}}$,

where $A_{131}=\left(\sqrt{A_{11}}-\sqrt{A_{33}}\right)^{2}$ is the Hamaker constant between microparticles in a fluid, and $z_{1}$ is the particle-particle separation distance.

The electrostatic force between a microparticle and the substrate in a fluid is given by $[15-17,37]$ : 

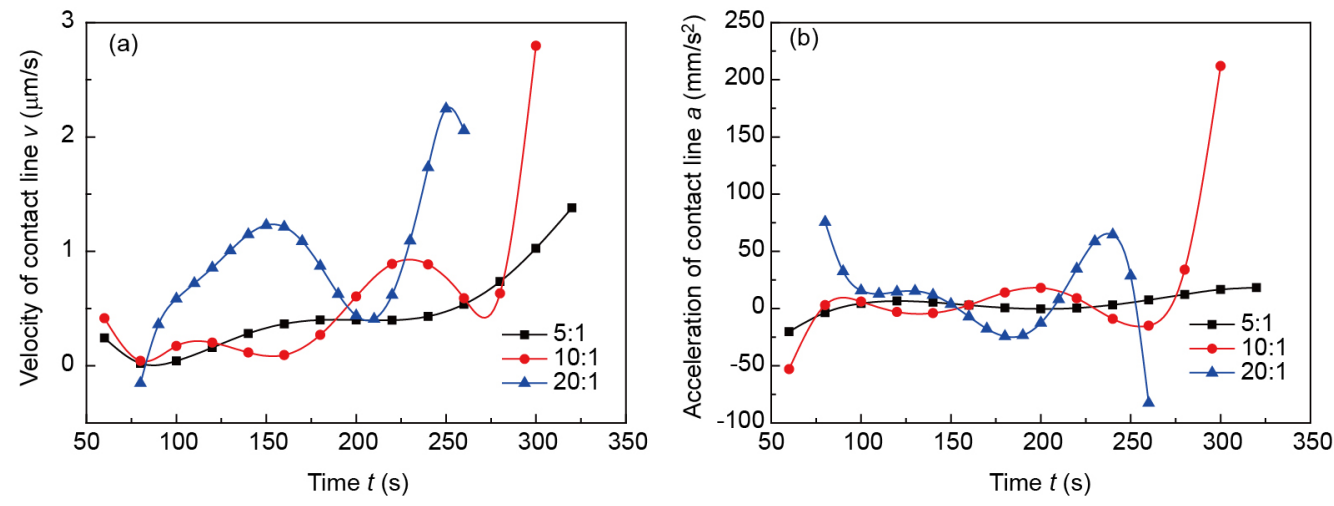

Figure 6 (Color online) Motion characteristics of depinning contact line. (a) Velocity; (b) acceleration.

$F_{\mathrm{eps}}=-2 R \varepsilon \kappa \frac{\left[\phi_{1}{ }^{2}+\phi_{2}{ }^{2}-2 \phi_{1} \phi_{2} \exp (\kappa z)\right]}{[\exp (2 \kappa z)-1]}$,

where $\varepsilon$ is the fluid permittivity, $\kappa$ is the reciprocal of Debye length, $\phi_{1}$ and $\phi_{2}$ are the surface potentials for microparticles and for the substrate, respectively.

The electrostatic force between microparticles in a fluid is given by [15-17,37]:

$F_{\text {epp }}=\frac{2 R \varepsilon \kappa \phi_{1}{ }^{2}}{\exp \left(\kappa z_{1}\right)+1}$.

The drag force acting on a microparticle is given by [15-17]
$F_{\mathrm{d}}=6 \pi R \eta v$,

where $\eta$ is the dynamic viscosity of the fluid and $v$ is the velocity of the evaporative flow.

Table 1 lists the parameters for force calculation and the values of some interaction forces acting on microparticles. Most of them are of orders of several or hundred piconewton, which are 8-12 orders higher than that of the inertial force $\sum F$, indicating that microparticles at the contact line are nearly in force equilibrium. To ensure such a quasi-static process, we suppose that microparticles near the liquid-vapor interface experience a capillary force, $F_{\mathrm{S}}$, given by $[3,23,24]$

Table 1 Parameters used in force calculations and interaction forces acting on microparticles

\begin{tabular}{|c|c|c|c|}
\hline Symbol & Physical Parameter & Value & Unit \\
\hline$A_{11}$ & Hamaker constant of PS microparticle & $6.3 \times 10^{-20}[38]$ & $\mathrm{J}$ \\
\hline$A_{22}$ & Hamaker constant of PDMS & $4.4 \times 10^{-20}[38]$ & $\mathrm{J}$ \\
\hline$A_{33}$ & Hamaker constant of water & $3.7 \times 10^{-20}[39]$ & $\mathrm{J}$ \\
\hline$A_{132}$ & $\begin{array}{l}\text { Hamaker constant between the PS microparticle } \\
\text { and PDMS in water }\end{array}$ & $0.10 \times 10^{-20}$ & $\mathrm{~J}$ \\
\hline$A_{131}$ & $\begin{array}{l}\text { Hamaker constant between PS microparticles } \\
\text { in water }\end{array}$ & $0.34 \times 10^{-20}$ & $\mathrm{~J}$ \\
\hline$\gamma_{1 \mathrm{v}}$ & surface tension of water & 0.072 & $\mathrm{~N} / \mathrm{m}$ \\
\hline$z$ & minimum separation distance & $0.4 \times 10^{-9}[16]$ & $\mathrm{m}$ \\
\hline$\varepsilon$ & permittivity of water & $7 \times 10^{-10}$ & $\mathrm{~F} / \mathrm{m}$ \\
\hline$\phi_{1}$ & surface potential of PS & $-59.5[40]$ & $\mathrm{mV}$ \\
\hline$\phi_{2}$ & surface potential of PDMS & $-45[41]$ & $\mathrm{mV}$ \\
\hline$\eta$ & dynamic viscosity of water & 0.0009 & $\mathrm{~Pa} \cdot \mathrm{s}$ \\
\hline$v$ & velocity of water in evaporating droplet & 1 & $\mu \mathrm{m} / \mathrm{s}$ \\
\hline$\kappa$ & reciprocal of the Debye length & $\left(430 \times 10^{-9}\right)^{-1}[16]$ & $\mathrm{m}^{-1}$ \\
\hline$F_{\text {wps }}$ & microparticle-substrate van der Waals fore & 520.83 & $\mathrm{pN}$ \\
\hline$F_{\text {eps }}$ & microparticle-substrate electrostatic fore & -179.44 & $\mathrm{pN}$ \\
\hline$F_{\mathrm{d}}$ & drag force & $8.48 \times 10^{-3}$ & $\mathrm{pN}$ \\
\hline
\end{tabular}


$F_{\mathrm{S}}=2 \pi R \gamma_{\mathrm{lv}} \cos \phi$,

where $\gamma_{\mathrm{lv}}$ is the liquid-vapor interfacial tension, $\phi$ is a parameter to be determined and $(\pi-2 \phi)$ is the angle of the liquid layer covering the outmost microparticle, as shown in Figure 7. The vertical component of this force will result in a positive or negative addition to normal force when the contact angle is less or larger than $90^{\circ}$. And the parallel component of this force will pull the microparticle towards the center of the solid-liquid interface.

Thus the total resultant force acting between the outmost microparticle and the substrate due to van der Waals and electrostatic interactions can be calculated by summing up the van der Waals, electrostatic, net gravitation and capillary forces:

$F_{\mathrm{a}}=F_{\text {wps }}+F_{\text {eps }}+F_{\mathrm{g}}+F_{\mathrm{s}} \cos \theta$

where $F_{\mathrm{g}}$ is the net gravitational force and $\theta$ is the contact angle. Because the density of PS microparticle is slightly larger than that of water, the net gravitational force can be ignored. The attractive force acting between the inner microparticle near the outmost one and the substrate can be written as:

$F_{\mathrm{a} 1}=F_{\mathrm{wps}}+F_{\mathrm{eps}}$.

The angle $\phi$ was determined by balancing all the forces along the radial direction as:

$F_{\mathrm{S}} \sin \theta-\left[f\left(F_{\mathrm{s}} \cos \theta+n F_{\text {wps }}+n F_{\text {eps }}\right)+n F_{\mathrm{d}}\right]=0$,

where $f$ is the kinetic friction between the microparticles and the substrate in a fluid. The first term in eq. (10) and all the terms in the square brackets are the driving and resistance forces, respectively.

As our earlier studies [28,30] showed, the angle $\theta$ ranges from nearly $90^{\circ}$ to about $50^{\circ}$ at the depinning contact line stage. Based on the above analysis and supposing that the friction coefficient $f$ is 0.1 , the critical angle $\phi_{\mathrm{C}}$ in different cases is calculated, as shown in Figure 8, indicating that i) there is only a very thin liquid layer acting on these microparticles because the angle $\left(180^{\circ}-2 \phi_{\mathrm{C}}\right)$ is far less than $1^{\circ}$, ii) both contact angle and the number of microparticles at the

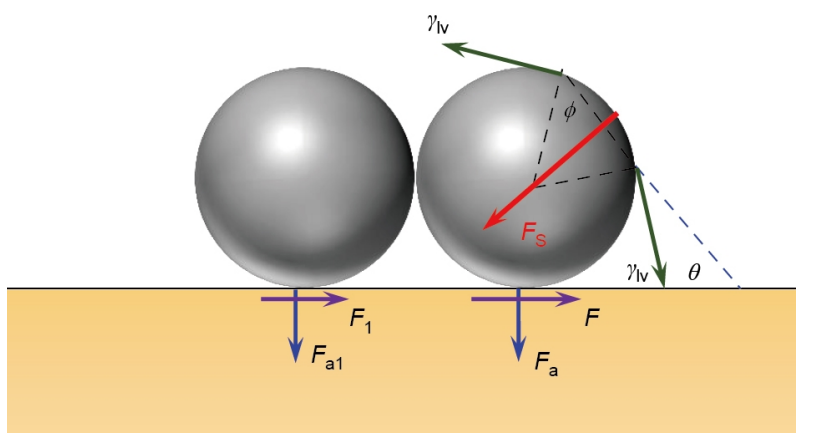

Figure 7 (Color online) Schematic of forces acting on microparticles near the contact line.

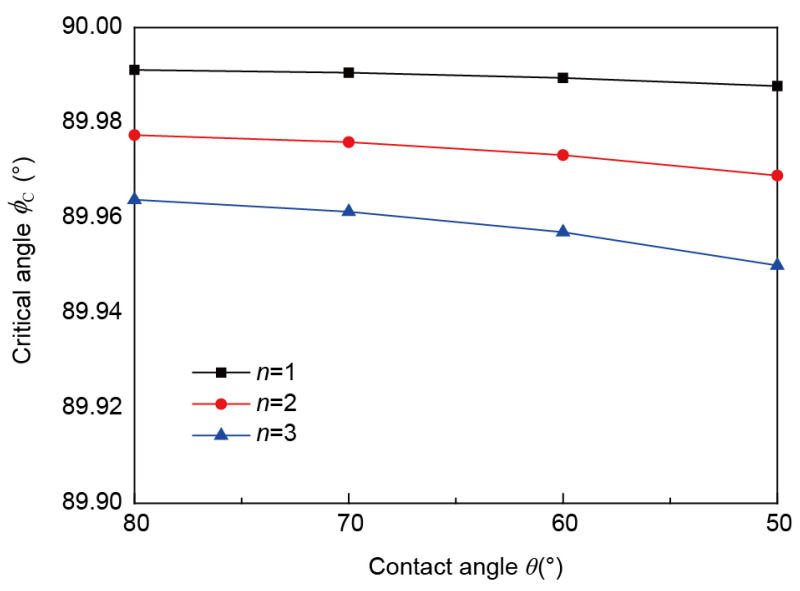

Figure 8 (Color online) Dependence of critical angle $\phi_{C}$ on contact angle and microparticle number.

contact line along the radial direction have little influence on $\phi_{\mathrm{C}}$, iii) since all the above forces are $\sim K R(K$ is a parameter of the unit of $\mathrm{N} / \mathrm{m}$ and independent of $R$ ), the critical angle $\phi_{\mathrm{C}}$ will be independent of the radius of microparticle, iv) the actual angle $\phi$ can only be slightly different from the critical value, if not, the acceleration of the microparticles would be very large, which did not consist with the experimental observation. Moreover, if the actual angle is slightly smaller than $\phi_{\mathrm{C}}$, then there will be a resulting force acting on the microparticles and pulling them towards the droplet. If the actual angle is slightly larger than $\phi_{C}$, then there will be a resulting force acting on the microparticles and driving them away from the droplet. Besides, when microparticles are self-pinned at the pinned contact line, eq. (9) is still valid, and it should be noted that the friction coefficient $f$ is no more than that of static friction.

Besides, since the inner microparticles near the outmost one are also quasi-static, the force balance can be given as:

$(n-i+1) f F_{\mathrm{a} 1}-F_{\text {wpp }}-F_{\text {epp }}=0$,

where $i$ denotes the $i$-th microparticle located near the contact line along the radial direction. $i=1$ denotes the outmost microparticle. Combining eq. (11) with eqs. (3), (5), and (9), we can obtain the minimum separation distance between microparticles.

\section{Conclusions}

Evaporation of sessile water droplets containing fluorescent PS microparticles was studied using PTV technique. It was found that some microparticles moved towards the contact line and were pinned at the pinned contact line, and later the microparticles located at the line moved together with the depinning contact line. Using the least square method, the moving distance of the contact line was obtained and then 
the motion characteristics of both the depinning contact line and microparticles located at the line were revealed. The results showed that the velocity and the maximum acceleration of these microparticles were, respectively, of the order of $1 \mu \mathrm{m} / \mathrm{s}$ and $20-200 \mathrm{~nm} / \mathrm{s}^{2}$, indicating their quasi-static motion. Finally, from the view of force equilibrium, we set up a new self-pinning mechanism of microparticles confined at a contact line and find that there is only a very thin liquid layer acting on these microparticles.

This work was supported by the National Natural Science Foundation of China (Grant Nos. 11572114, 11572335, and U1562105), the Opening Fund of State Key Laboratory of Nonlinear Mechanics (LNM), the CAS Strategic Priority Research Program (Grant No. XDB22040403), and the CAS Key Research Program of Frontier Sciences (Grant No. QYZDJ-SSW-JSC019).

1 R. D. Deegan, O. Bakajin, T. F. Dupont, G. Huber, S. R. Nagel, and T. A. Witten, Nature 389, 827 (1997).

2 H. Hu, and R. G. Larson, J. Phys. Chem. B 110, 7090 (2006).

3 Y. P. Zhao, Physical Mechanics of Surfaces and Interfaces (Science Press, Beijing, 2012).

4 U. Thiele, Adv. Colloid Interf. Sci. 206, 399 (2014).

5 P. J. Yunker, T. Still, M. A. Lohr, and A. G. Yodh, Nature 476, 308 (2011).

6 H. B. Eral, D. M. Augustine, M. H. G. Duits, and F. Mugele, Soft Matter 7, 4954 (2011).

7 D. Mampallil, H. B. Eral, D. van den Ende, and F. Mugele, Soft Matter 8, 10614 (2012).

8 T. Still, P. J. Yunker, and A. G. Yodh, Langmuir 28, 4984 (2012).

9 L. Cui, J. Zhang, X. Zhang, L. Huang, Z. Wang, Y. Li, H. Gao, S. Zhu, T. Wang, and B. Yang, ACS Appl. Mater. Interf. 4, 2775 (2012).

10 A. Crivoi, and F. Duan, Langmuir 29, 12067 (2013).

11 M. Majumder, C. S. Rendall, J. A. Eukel, J. Y. L. Wang, N. Behabtu, C. L. Pint, T. Y. Liu, A. W. Orbaek, F. Mirri, J. Nam, A. R. Barron, R. H. Hauge, H. K. Schmidt, and M. Pasquali, J. Phys. Chem. B 116, 6536 (2012).

12 Y. F. Li, Y. J. Sheng, and H. K. Tsao, Langmuir 29, 7802 (2013).

13 Z. Q. Lin, Evaporative Self-assembly of Ordered Complex Structures (World Scientific Publishing Co. Pte. Ltd., Singapore, 2012).
14 B. M. Weon, and J. H. Je, Phys. Rev. E 82, 015305 (2010).

15 J. Jung, Y. W. Kim, J. Y. Yoo, J. Koo, and Y. T. Kang, Anal. Chem. 82, 784 (2010).

16 T. S. Wong, T. H. Chen, X. Shen, and C. M. Ho, Anal. Chem. 83, 1871 (2011).

17 V. H. Chhasatia, and Y. Sun, Soft Matter 7, 10135 (2011).

18 B. M. Weon, and J. H. Je, Phys. Rev. Lett. 110, 028303 (2013).

19 R. Bhardwaj, X. Fang, P. Somasundaran, and D. Attinger, Langmuir 26, 7833 (2010).

20 Q. Yuan, and Y. P. Zhao, J. Fluid. Mech. 716, 171 (2013).

21 Q. Yuan, X. Huang, and Y. P. Zhao, Phys. Fluids 26, 092104 (2014).

22 Y. Q. Li, H. A. Wu, and F. C. Wang, Langmuir 32, 12676 (2016).

23 A. S. Sangani, C. Lu, K. Su, and J. A. Schwarz, Phys. Rev. E 80, 011603 (2009).

24 F. Shao, T. W. Ng, J. Efthimiadis, A. Somers, and W. Schwalb, J. Colloid Interf. Sci. 377, 421 (2012)

25 Y. Zhao, Theor. Appl. Mech. Lett. 4, 034002 (2014).

26 Y. P. Zhao, Sci. China-Phys. Mech. Astron. 59, 114631 (2016).

27 F. C. Wang, and Y. P. Zhao, Soft Matter 7, 8628 (2011).

28 Y. S. Yu, Z. Wang, and Y. P. Zhao, J. Colloid Interf. Sci. 365, 254 (2012).

29 M. C. Lopes, and E. Bonaccurso, Soft Matter 8, 7875 (2012).

30 Y. S. Yu, Z. Q. Wang, and Y. P. Zhao, Acta Mech. Sin. 29, 799 (2013).

31 M. C. Lopes, and E. Bonaccurso, Soft Matter 9, 7942 (2013).

32 L. Chen, G. K. Auernhammer, and E. Bonaccurso, Soft Matter 7, 9084 (2011).

33 L. Chen, E. Bonaccurso, and M. E. R. Shanahan, Langmuir 29, 1893 (2013).

34 X. Zheng, G. P. Kong, and Z. H. Silber-Li, Acta Mech. Sin. 29, 411 (2013).

35 X. L. Xia, X. Zheng, X. F. Huang, J. Z. Zhou, and Y. S. Yu, Appl. Math. Mech 8, 495 (2017).

36 M. Elimelech, J. Gregory, X. Jia, and R. A. Williams, Particle Deposition \& Aggregation (Butterworth Heinemann Publications, Woburn, 1998), pp. 38-46.

37 Y. S. Yu, AIP Adv. 6, 095124 (2016).

38 C. J. Drummond, and D. Y. C. Chan, Langmuir 13, 3890 (1997).

39 A. Koh, G. Gillies, J. Gore, and B. R. Saunders, J. Colloid Interf. Sci. 227, 390 (2000).

40 A. Nakao, Y. Suzuki, and M. Iwaki, J. Colloid Interf. Sci. 197, 257 (1998).

41 B. J. Kirby, and E. F. Hasselbrink, Electrophoresis 25, 203 (2004). 